Open Access

\title{
SAMU: design and implementation of frequency selectivity-aware multi-user MIMO for WLANs
}

\author{
Yongjiu Du ${ }^{1}$, Yan Shi ${ }^{*}$ (D) Ehsan Aryafar ${ }^{3}$, Pengfei Cui ${ }^{4}$, Joseph Camp ${ }^{2}$ and Mung Chiang ${ }^{5}$
}

\begin{abstract}
The traffic demand of wireless networks is expected to increase 1000-fold over the next decade. In anticipation of such increasing data demand for dense networks with a large number of stations, IEEE 802.11 ax has introduced key technologies for capacity improvement including Orthogonal Frequency-Division Multiple Access (OFDMA), multi-user multi-input multi-output (MU-MIMO), and greater bandwidth. However, IEEE 802.11 ax has yet to fully define a specific scheduling framework, on which the throughput improvement of networks significantly depends. Even within a $20 \mathrm{MHz}$ of bandwidth, users experience heterogeneous channel orthogonality characteristics across sub-carriers, which prevents access points (APs) from achieving the ideal multi-user gain. Moreover, frequency selectivity increases as bandwidth scales and correspondingly severely deteriorates multi-user MIMO performance. In this work, we develop a novel channel adaptation scheme, named selectivity-aware multi-user MIMO (SAMU), to combat the issue of frequency selectivity and support coexistence among users in the network by jointly assigning subsets of sub-carriers to selected users and implementing downlink MU-MIMO. To do so, we first investigate the channel characteristics of an indoor environment. We then consider the frequency selectivity of current and emerging WiFi channel bandwidths to optimize multi-user MIMO by dividing the occupied sub-carrier resources into equally sized sub-channels according to the level of frequency selectivity. In our design, each sub-channel is allocated according to the largest bandwidth that can be considered frequency-flat, and an optimal subset of users is chosen to serve in each sub-channel according to spatial orthogonality. As a result, we support more simultaneous users than current 802.11 designs and achieve a significant performance improvement for all users in the network. Additionally, we propose a selectivity-aware high efficiency (SA-HE) mode, which is based on and fully backward compatible with the existing IEEE 802.11 ax standard. Finally, over emulated and real indoor channels, we show that SAMU can achieve as much as $84.8 \%$ throughput improvement compared to existing multi-user MIMO schemes in IEEE 802.11 ax.
\end{abstract}

Keywords: WiFi, IEEE 802.11ax, High-efficiency mode, Multi-user-MIMO, OFDMA, Frequency selectivity channel

\section{Introduction}

To address the exponentially increasing traffic demand in wireless networks, the WiFi community is currently developing IEEE 802.11ax to improve the efficiency of WiFi networks in dense scenarios $[1,2]$. Unlike 802.11ac, 802.11ax operates in both the 2.4 and $5 \mathrm{GHz}$ bands and employs OFDMA, a technological improvement that increases the aggregate network throughput and potential

*Correspondence: shiy@smu.edu

${ }^{2}$ Department of Electrical Engineering, Southern Methodist University, Dallas, TX, USA

Full list of author information is available at the end of the article user density while reducing latency by efficiently scaling resources for multiple users in both the frequency and time domains [3-5]. This multiple antenna technology is usually referred as MIMO [6]. Also, IEEE 802.11ax implements lower overhead for packet transmission at high rates and reduces sub-carrier spacing to improve robustness to frequency selective interference. The IEEE 802.ax standard supports downlink MU-MIMO and also provides uplink MU-MIMO feasibility [7]. MU-MIMO uses channel feedback from users to carefully align the phases of each transmission so that users can coexist on the same channel bandwidth. Each user can correctly receive packets simultaneously due to spatial diversity 
and channel pre-coding by the transmitter. The total throughput, however, highly depends on the relationship between the channel responses of all users. The AP attempts to select an optimal subset of users that maximizes the overall spatial efficiency. Optimal user selection is usually based on the traffic demands of and channel characteristics experienced by all the users. However, with frequency selectivity, a subset of users may have good spatial reuse on some carrier frequencies due to the orthogonality of the sub-channels experienced by each user, but poor orthogonality on other frequencies due to the frequency diversity across the channel bandwidth. The critical enhancements of implementing OFDMA and MU-MIMO from the latest IEEE 802.11ax draft standard are known to independently improve the system capacity. However, to the best of our knowledge, the capacity achievable via their joint use is an open question. In addition, there is currently no such scheme to adapt the bandwidth of MU-MIMO WiFi according to the frequency selectivity of the channel.

Due to the increasing sampling and operating speeds of electronic devices, with the evolution of various wireless standards, modern wireless systems tend to use more and more bandwidth to improve the system throughput. For example, IEEE 802.11a uses up to $20 \mathrm{MHz}$, IEEE 802.11n uses up to $40 \mathrm{MHz}$, IEEE 802.11ac uses up to $160 \mathrm{MHz}$, and IEEE 802.11ad uses multiple $\mathrm{GHz}$ of available bandwidth at a $60-\mathrm{GHz}$ carrier frequency. 802.11ax standards have recently sought to increase the total bandwidth using channel bonding, an advanced physical layer technique that allows combining several non-continuous channels to provide high transmission rate [7]. Thus, the signal bandwidth is only expected to increase for emerging WiFi networks.

Even for typical environmental settings for WiFi networks, which seem relatively simple, reflections from surrounding objects cause strong multi-path effects from sender to receiver. As a result, frequency-selective fading occurs across the channel bandwidth, where carrier frequencies experience diverse received signal levels. Narrow-band wireless systems are mostly immune to such frequency-selective fading. However, for systems with much wider bandwidth (e.g., at least $20 \mathrm{MHz}$ ), frequencyselective fading can become a dominant channel effect, degrading the system performance. Frequency selectivity ${ }^{1}$ becomes an even more critical issue for MU-MIMO systems, severely degrading the high throughput previously anticipated [8-10].

In this work, we consider the frequency selectivity to divide the existing downlink 802.11 channels into smaller sub-channels (including division granularity comparable to an 802.11ax resource unit) and dynamically allocate the maximum width appropriate for frequencyflat sub-channels. In doing so, the channel response per sub-channel can be regarded as homogeneous for all frequencies. Since each sub-channel has different frequency characteristics, we develop an effective algorithm to choose a corresponding optimal subset of users for each sub-channel by taking into account both frequency diversity and interference. The subset of users across different sub-channels could be the same, partially overlapped, or mutually exclusive, depending on the channel characteristics of all users and the degree to which frequency selectivity occurs. Hence, our selectivityaware multi-user MIMO scheme, SAMU, overcomes a key issue in wideband WiFi networks: frequency selectivity. Moreover, we propose a selectivity-aware high efficiency (SA-HE) mode, which is based on an extension to the latest IEEE 802.11ax amendments. We experimentally evaluate SAMU over emulated channels for repeatability and control as well as in the field for representative frequency-selective scenarios. Lastly, SAMU can support a larger number of simultaneous users than the current WiFi design. Since the entire channel is divided into multiple sub-channels, a separate subset of users is served on each sub-channel. In a wide range of system configurations across emulated and real indoor channels, we show that SAMU can significantly improve the system throughput by $84.8 \%$.

The main contributions of this paper are as follows:

1 We measure and evaluate the channel response characteristics and channel orthogonality on each sub-carrier for MU-MIMO scenarios in indoor environments, showing that frequency selectivity not only applies to a single link but also affects the user selections for MU-MIMO.

2 We develop protocols and algorithms for a selectivity-aware MU-MIMO scheme, SAMU, which considers the degree to which frequency selectivity is present on the channel, allocates frequency-flat sub-channels, and chooses an optimal subset of users for each sub-channel to improve the spatial efficiency of the network.

3 We present the SAMU design which spans physical (PHY) and media access control (MAC) layer modifications to support sub-channel and user selection. Specifically, we outline a SA-HE mode as an extension to the latest IEEE 802.11ax draft 2.0.

4 We evaluate a sub-channel division adaptation scheme to dynamically change the number of sub-channels according to the delay spread in environment of operation.

5 We conduct experiments with programmable hardware devices over repeatable and realistic channel conditions on a channel emulator and in a representative environment, showing significant throughput improvements. 
The rest of the paper is organized as follows. We provide related work as well as background knowledge in Section 2. In Section 3, we discuss frequency diversity and channel orthogonality for MU-MIMO schemes in indoor environments. Then, we propose our physical and media access layer design for SAMU in Section 4. In Section 5, we introduce the hardware setup for SAMU evaluation. In Section 6, we leverage our delay spread measurements for both 2.4 and $5.2 \mathrm{GHz}$ and implement sub-channel division adaptation on diverse channels. Then, we experimentally evaluate our design. Finally, in Section 7, we conclude our work.

\section{Related work}

This work is closely related to the following areas: (i) MU-MIMO, (ii) channel bonding, (iii) frequency diversity measurements in indoor environments, and (iv) frequency-aware wireless communication schemes.

\subsection{MU-MIMO}

One of the key features in IEEE 802.11ax standard is the enhanced MU-MIMO which obtains multiplexing gains by multiple antennas [7] and provides uplink MU-MIMO feasibility. In the latter case, the transmitter sends the controlling and synchronization frames to provide reception from multiple users [1]. Other MIMO schemes include beamforming, spatial diversity, and spatial multiplexing. Multi-user beamforming can achieve additional throughput improvement by properly setting the steering matrix of the transmitting links to enable parallel data transmissions $[10,11]$. Beamforming can improve the received signal strength of the intended user and reduce interference to unintended users [12, 13]. Spatial diversity can improve the transmission stability and throughput, while spatial multiplexing aims to improve the system throughput by sharing the channel via multiple parallel transmissions [14]. However, these works either use narrow-band signals or are evaluated in wireless channels with minimal frequency selectivity. In contrast, we show that multi-user systems in an indoor environment can be significantly affected by frequency selectivity.

\subsection{Channel bonding}

Non-continuous channel bonding is supported in IEEE 802.11ax, enabling devices to simultaneously transmit data by combining non-continuous channels [3]. The main task of non-continuous channel bonding is to select available channels for transmission [7]. With channel bonding, multiple $20 \mathrm{MHz}$ channels can be bonded together to form a wide channel. However, even with $20 \mathrm{MHz}$ of bandwidth, users experience heterogeneous channel orthogonality characteristics across sub-carriers, which prevents access points from achieving the ideal multi-user gain. For systems with much wider bandwidth via channel bonding, frequency-selective fading can become a dominant effect on the system performance, significantly degrading the throughput benefits brought by bandwidth expansion. Although our experiments are conducted on a $20 \mathrm{MHz}$ bandwidth due to hardware limitations, we demonstrate that the system performance can be further improved by channel division comprehensively over a wider channel (bonding of several continuous channels) or separately over several non-continuous channel (bonding of several non-continuous channels).

\subsection{Frequency diversity measurements in indoor environments}

In order to achieve high levels of capacity and reliability in wireless networks, measuring the wireless channel is critical for designing and implementing effective systems. Frequency selectivity and time selectivity are the two key wireless channel effects that each pose a challenge to system designers. Frequency selectivity typically corresponds to the multi-tap effect, while time selectivity typically corresponds to channel fading. Channel measurements have been done in different environments and frequency bands [15-21]. In [15], the authors measure the channel heterogeneity and temporal stability to guide their system design. In [16], the authors investigated the temporal, spatial, and spectral fading in home and office environments on the $5.2 \mathrm{GHz}$ band. Power-delay profile characteristics are measured in [18]. In [17, 20], channel characteristics of millimeter wave signals are measured to study the feasibility of next generation wireless networks in that band. In contrast to these single antenna/user works, we study the impact of frequency diversity on multi-user MIMO scenarios.

Frequency-aware wireless communication: For either indoor or outdoor environments, significant frequency diversity has been observed. Traditional wireless protocols use the same settings on all of the frequencies or subcarriers across the entire channel bandwidth, regardless of the channel gain diversity. Applying different configurations on sub-carriers according to their respective channel gains can improve the system performance. Several prior works have developed systems dealing with frequency diversity [15, 22-24]. In [22], the author measured per sub-carrier SNR to enable the transmitter to adapt the bit rate independently across different sub-carriers. The authors in [15] offer proportional protection of symbols according to their channel quality and importance level. In [23], an effective algorithm was developed to select the appropriate spectrum to use for each transmission by taking into account both frequency diversity and interference. In [24], a system was developed to redistribute the transmit power across the sub-carriers to better cope with the frequency selectivity, subject to a fixed power budget constraint. In 3GPP-LTE, frequency-aware user 
scheduling and resource allocation have also been shown to provide significant performance improvement [25]. In contrast, we consider both spatial and spectral dimensions for user selection in a MU-MIMO scenario and perform sub-channel allocation according to the level of frequency selectivity to improve MU-MIMO performance. As a result, the abovementioned related work (e.g., $[15,24,25])$ can be applied in our proposed SAMU to obtain an aggregate performance improvement. In this paper, we focus on showing the performance improvement without adopting further possible enhancements.

\section{Impact of frequency selectivity on MU-MIMO performance}

In this section, we consider the impact of frequency diversity on MU-MIMO performance. We first examine the extent of frequency selectivity in indoor environments. Then, we measure the impact of frequency selectivity on channel orthogonality of different users. Finally, we show how selectivity-aware user selection can benefit MU-MIMO performance.

\subsection{Frequency diversity in indoor environments}

In a given wireless environment, there often exists a large number of reflections and refraction from obstacles that cause the number of signal paths to approach infinity. In order to model the channel, these paths can be abstracted into groups based on their attenuation and delay from the first-arriving signal. Each group is referred to as a channel tap. Within each group, due to the different phases and amplitudes generated by the different path lengths, channel gain fluctuations are generated. The channel taps are then aggregated into a single channel model (i.e., a multitap channel model) which represents a given environment with each tap having a fading characterization based on a certain probability distribution (e.g., Rayleigh or Rician).

One standard metric to quantify the multi-path richness of a communication channel is delay spread, which is directly related to frequency selectivity in the frequency domain [14]. More formally, we define the delay spread as $\tau$, which is the time difference between the first qualified multi-path channel tap and the last qualified multi-path channel tap. A qualified channel tap should have a power greater than $P_{\max }-\eta$, in which $P_{\max }$ is the power of the strongest channel tap, and $\eta$ is a constant value. This model is depicted in Fig. 1. Let $P(\tau)$ denote the powerdelay profile of the channel. Then, the RMS delay spread of the channel is given by:

$$
\tau_{\mathrm{rms}}=\sqrt{\frac{\int_{0}^{\infty}(\tau-\bar{\tau})^{2} P(\tau) d \tau}{\int_{0}^{\infty} P(\tau) d \tau}}
$$

Here, $\bar{\tau}$ is the mean delay spread of the channel and expressed as:

$$
\bar{\tau}=\frac{\int_{0}^{\infty} \tau P(\tau) d \tau}{\int_{0}^{\infty} P(\tau) d \tau}
$$

We conduct experiments to measure delay spread and channel characteristics of an office indoor environment using WARP [26]. WARP is a software-defined radio platform with extensive over-the-air experimentation which enables the users to implement custom software codes. In order to achieve channel sounding, we generate training OFDM symbols with 256 sub-carriers as the channel probe signals, known as the IEEE 802.11ax null data packet announcement (NDPA) frame [1]. The probe signals are followed by a trigger signal. The channel probe signals and the trigger signal are then transferred to the

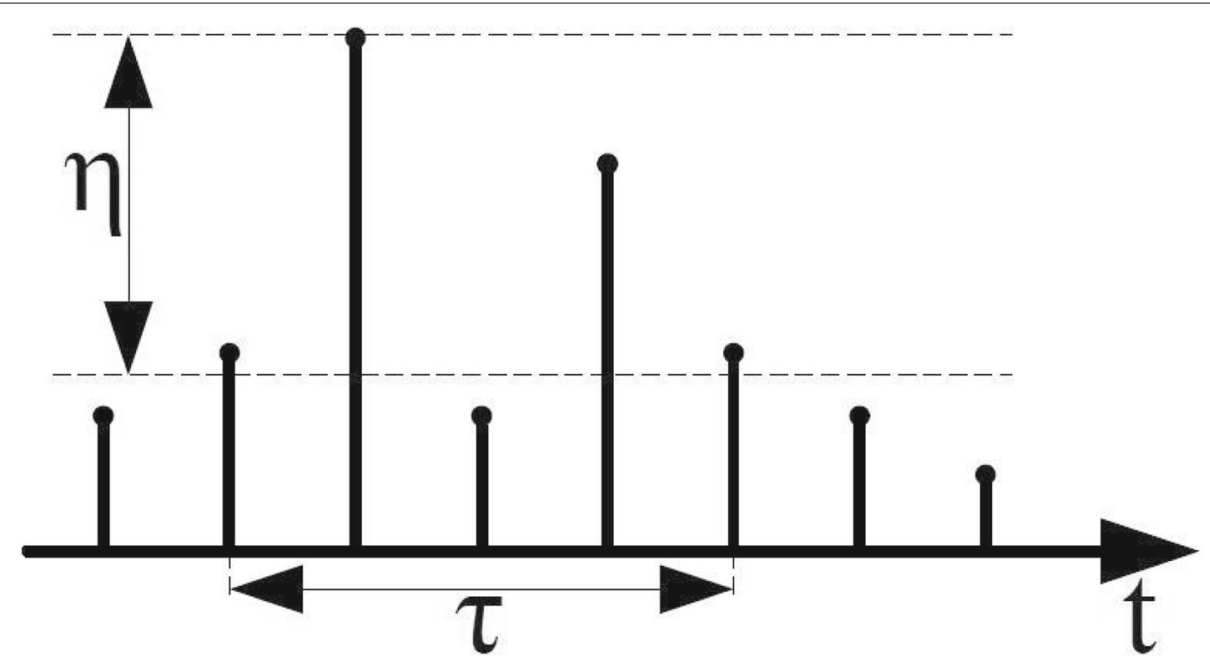

Fig. 1 Delay spread measurement model 
transmitting WARP board via an Ethernet cable. WARP is then triggered to transmit data samples over the air. The receiver samples the received signals and then transfers the raw samples to a $\mathrm{PC}$, where the channel characteristics are estimated. Note that the channel sounding procedure needs to be performed periodically due to channel fluctuations. Our experiments are conducted on the 2.4 and $5.2 \mathrm{GHz}$ band over a $20 \mathrm{MHz}$ bandwidth. For practical implementations, delay spread can be measured at the receiver and fed back to the transmitter along with channel information.

Figures 2a and 3a depict the CDF of the measured delay spread for different $\eta$ values at 2.4 and $5.2 \mathrm{GHz}$, respectively. It is interesting to observe that $2.4 \mathrm{GHz}$ suffers from larger multi-path effects versus $5.2 \mathrm{GHz}$. For example, the maximum delay spread is approximately between 50 and $260 \mathrm{~ns}$ at $5.2 \mathrm{GHz}$, corresponding to a difference in path lengths that the multi-path signals traverse of approximately 15 to $78 \mathrm{~m}$. In contrast, the maximum delay spread can reach up to $300 \mathrm{~ns}$ at $2.4 \mathrm{GHz}$ in the same environment, corresponding to a maximum path signals traverse of $90 \mathrm{~m}$.

Due to the multi-path components in the environment, signals experience different levels of channel gain on different frequencies. Figures $2 \mathrm{~b}$ and $3 \mathrm{~b}$ depict the measured channel gain difference across all sub-carriers. We observe that, over different sub-carriers, the channel gain diversity can be as much as 50 and $55 \mathrm{~dB}$ for 2.4 and $5.2 \mathrm{GHz}$, respectively, while the average gain diversity is around $31 \mathrm{~dB}$

The phase distortion of the channel also plays a key role in MU-MIMO. We now measure the channel phase difference across all the sub-carriers in the same indoor environment and show the results in Figs. $2 \mathrm{c}$ and $3 \mathrm{c}$ for 2.4 and $5.2 \mathrm{GHz}$, respectively. We observe that, for most of the channel implementations, the maximum phase difference across all the sub-carriers approaches $2 \pi$ for both frequency bands. We conclude that, even at a $20 \mathrm{MHz}$ of bandwidth, users can experience heterogeneous frequency selectivity, with $2.4 \mathrm{GHz}$ presenting a relatively larger effect on channel response characteristics than $5.2 \mathrm{GHz}$.

\subsection{MIMO channel orthogonality}

Channel orthogonality is a metric to evaluate the potential spatial efficiency of MU-MIMO systems, which can be measured by the following equation [27]:

$$
\alpha_{i, j, k}=1-\frac{\left|\boldsymbol{h}_{i, k} \boldsymbol{h}_{j, k}^{*}\right|}{\left\|\boldsymbol{h}_{i, k}|| \mid \boldsymbol{h}_{j, k}\right\|}
$$

The vectors $\boldsymbol{h}_{i, k}$ and $\boldsymbol{h}_{j, k}$ are channel responses from the AP to user $i$ and user $j$ on sub-carrier $k$, respectively. $\alpha_{i, j, k}$ has a range of $(0,1)$. For $\alpha_{i, j, k}$, a value of 0 denotes that the two channel vectors are fully parallel, while a value of 1 denotes being fully orthogonal. The more orthogonal the vectors, the less one user interferes with another user. Hence, better spatial efficiency can be achieved to improve the throughput.

For different users, if their channel vectors to the AP are orthogonal, optimal multi-user gain can be achieved. However, in practice, it is hard to find a set of users whose channel vectors are fully orthogonal, especially for a small number of users. An exhaustive search over the entire user set can be used to find the optimal selected simultaneous user set from a small number of total users [6, 28]. However, exhaustive search is not time-efficient. Instead, a semi-orthogonal user selection algorithm has been developed, which is shown to be sub-optimal. For a large number of users, semi-orthogonal user selection provides a sub-optimal performance with lower-time computational complexity [29].

In existing IEEE 802.11 MU-MIMO schemes, each user occupies the entire channel bandwidth. The signal from each user could be correctly decoded leveraging the spatial diversity of the wireless channel. For the AP to maximize the overall spatial efficiency, the selection of such optimal subset of users depends on the channel characteristics experienced by and the traffic demands of all the
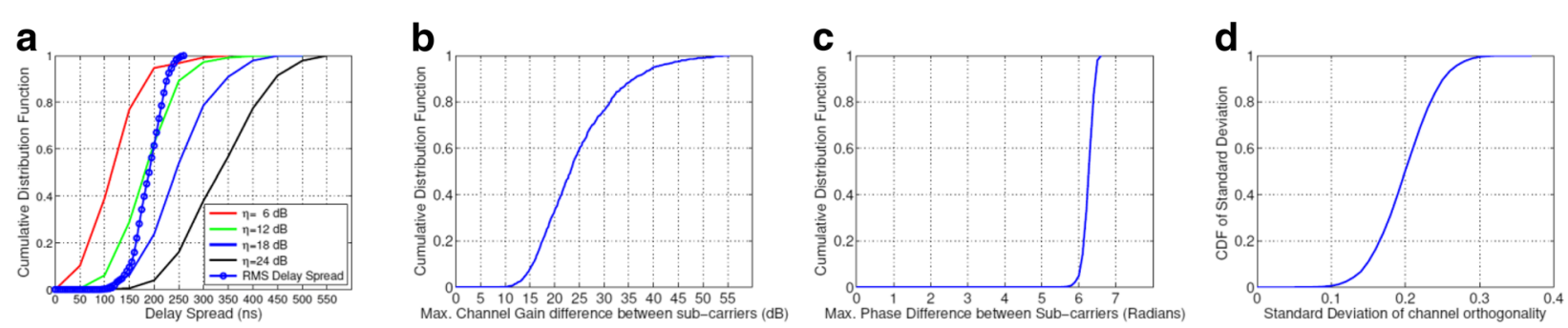

Fig. 2 Verifying impact of frequency selectivity on MU-MIMO performance on $2.4 \mathrm{GHz}$ : a delay spread in an office environment, $\mathbf{b}$ maximum channel gain difference across all sub-carriers in an office environment, $\mathbf{c}$ maximum channel phase difference across all sub-carriers in an office environment, $\mathbf{d}$ channel orthogonality on different sub-carriers between 2 users 

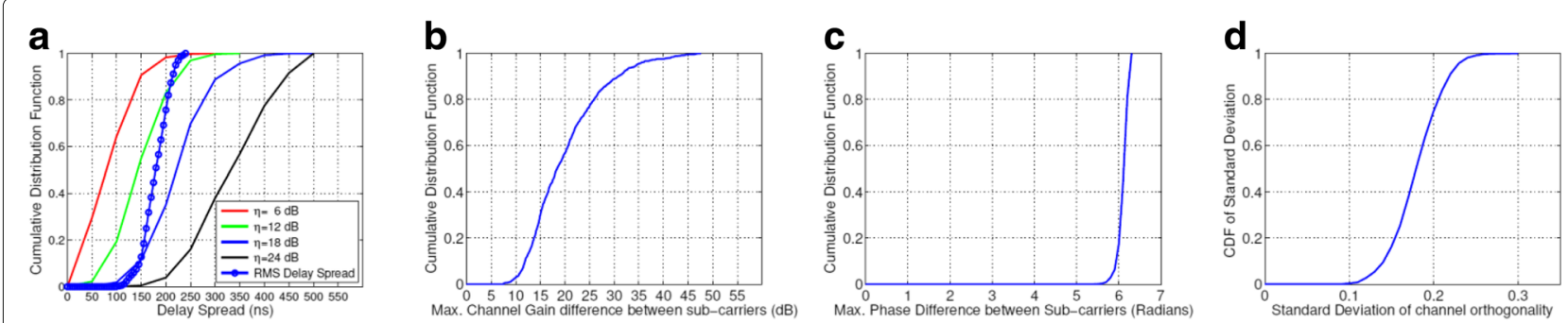

Fig. 3 Verifying impact of frequency selectivity on MU-MIMO performance on $5.2 \mathrm{GHz}$ : a delay spread in an office environment, b maximum channel gain difference across all sub-carriers in an office environment, $\mathbf{c}$ maximum channel phase difference across all sub-carriers in an office environment, $\mathbf{d}$ channel orthogonality on different sub-carriers between 2 users

users. However, with frequency selectivity in the channel, a subset of users may perform better spatial efficiency if only some sub-carriers are used due to the orthogonality of the corresponding sub-channels. However, the same scheme may not be optimal for other sub-carriers due to the frequency selectivity changing the sub-channel orthogonality. The problem is exacerbated with wider bandwidths.

We conduct experiments to quantify the level of channel orthogonality in indoor environments. We use one WARP board as the AP and two other WARP boards as users. The AP is equipped with 4 antennas, while each user has only 1 antenna. We set the number of sub-carriers to 256 . We show the measured channel orthogonality between the two users on different sub-carriers in Figs. $2 \mathrm{~d}$ and $3 \mathrm{~d}$. We can see that, due to frequency-selective fading, the channel orthogonality of the users fluctuate significantly across different sub-carriers for both frequency bands, with a standard deviation ranging from around 0.10 to 0.3 . Also, $2.4 \mathrm{GHz}$ has presented a relatively larger variance in channel orthogonality than $5.2 \mathrm{GHz}$, with the maximum standard deviation approaching 0.34 . For the sub-carriers that possess less orthogonality, spatial efficiency degrades significantly.

\subsection{Selectivity-aware user selection}

In existing MU-MIMO WiFi systems, the AP selects the optimal group of MU-MIMO users based on an average expected performance across the entire channel. This works well for channels with the same frequency response across the whole bandwidth (i.e., for flat-fading channels). However, as we have shown experimentally, in most wireless communication scenarios (even indoor), the multipath effect causes frequency selectivity. As a result, the selected subset of users could be optimal for part of the channel bandwidth, but not optimal for other parts of the channel bandwidth.

If we divide the whole bandwidth into a number of chunks based on channel coherence bandwidth, for each chunk, users experience similar channel effects. However, for different chunks, users experience different channel effects due to frequency selectivity, and the system chooses different subset of users to serve accordingly. In our indoor experiments described in Section 6, the frequency chunk has an approximate bandwidth of $500 \mathrm{kHz}$. Assume that $n$ denotes the number of chunks divided for the current bandwidth. Thus, $n$ with a value of 40 for a $20 \mathrm{MHz}$ of bandwidth means that we divide the whole bandwidth into 40 frequency-flat regions. As the total bandwidth increases, $n$ will increase proportionally. Therefore, in our proposed system, SAMU, we divide the given bandwidth into sub-channels and select the optimal set of users for each sub-channel. The number of these sub-channels, $n$, depends on both the bandwidth, the frequency selectivity characteristics of the environment across the given bandwidth (the maximum delay spread), and the frame duration. The set of users across different sub-channels could be the same, partially overlap, or be mutually exclusive.

For a given sub-carrier, different pairs of users experience different levels of channel orthogonality. One pair of users may experience poor orthogonality, while another pair of users may have good orthogonality. If we choose the users on a sub-carrier basis or group of sub-carriers basis, we can get optimal orthogonality on each sub-carrier. Therefore, throughput could be potentially improved. Thus, with intelligent selection of users and channel partitioning, MU-MIMO performance can be improved significantly. We show a simple example of the channel division for two pairs of users on different sub-carriers in Fig. 4. One critical change with 802.11ax is that the sub-carrier spacing has been reduced to one fourth of the previous 802.11 revisions, which means that the FFT size proposed for a $20 \mathrm{MHz}$ of bandwidth is 256 . In this scenario, if we divide the entire $20-\mathrm{MHz}$ bandwidth into 4 sub-channels, then there will be 64 sub-carriers in each sub-channel. For sub-channels 1, 2, 3, and 4, we select the user groups of $\{2,3\},\{1,2\},\{2,3\},\{1,2\}$ based on users' 


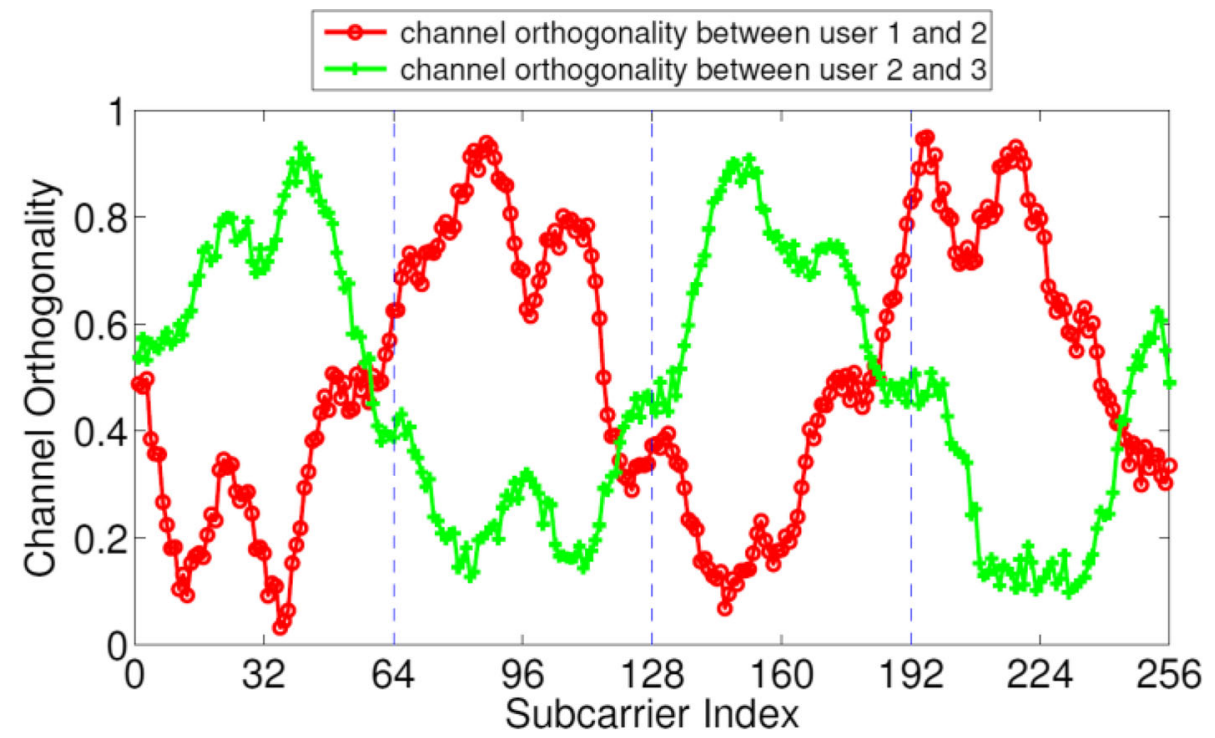

Fig. 4 Demonstration of selectivity-aware channel division on $5.2 \mathrm{GHz}$

internal channel orthogonality, respectively. As a result, there will be significant throughput improvement, as we will see in Section 6.

\section{Design of SAMU}

In this section, we describe the design of our selectivityaware MU-MIMO system, SAMU, for wideband WiFi. In particular, SAMU consists of $(i)$ a PHY layer design that supports sub-channel encoding, (ii) a MAC layer design to optimally select users in a given sub-channel, (iii) an ACK management mechanism for multiple data packets across the channel bandwidth, and (iv) an adaptation mechanism for sub-channel division according to diverse delay spreads across environments. We first describe the physical layer aspects of SAMU and outline a SA-HE mode which would be an extension to IEEE 802.11ax. We then discuss the media access layer aspects of SAMU and propose a selectivity-aware user selection algorithm. Finally, we propose bandwidth division and adaptation algorithms that adjust SAMU's operation based on changes in the environment.

\subsection{Physical layer signaling}

Figure 5a shows the HE mode data frame format, known as the high efficiency multi-user physical protocol data unit (HE MU PPDU) for transmitting to one or more users, defined in the IEEE 802.11ax standard. The 802.11ax frame, similar to that of 802.11ac, starts with the legacy (non-HE) preamble. The L-STF, L-LTF, and L-SIG fields in the legacy preamble are used for backward compatibility and coexistence with prior IEEE 802.11 standards (e.g., IEEE $802.11 \mathrm{a} / \mathrm{g} / \mathrm{n} / \mathrm{ac}$ ). These fields are used for frame detection, automatic gain control (AGC) settings, channel estimation, and frequency/timing synchronization. The HE preamble includes RL-SIG, HE-SIG-A, HESIG-B, HE-STF, and HE-LTF portion. The RL-SIG field is another symbol repetition of L-SIG for the early detection of 802.11ax frames. The HE-SIG-A field is used to convey the common control information, including the bandwidth, guard interval (GI), space-time block coding (STBC), basic service set (BSS), and transmission opportunity (TXOP) duration. The HE-SIG-B field is present in 802.11ax only for MU-MIMO operations, which is

a

\begin{tabular}{|c|c|c|c|c|c|c|c|c|c|c|}
\hline L-STF & L-LTF & L-SIG & RL-SIG & $\begin{array}{c}\text { HE- } \\
\text { SIG-A }\end{array}$ & $\begin{array}{c}\text { HE- } \\
\text { SIG-B }\end{array}$ & $\begin{array}{c}\text { HE- } \\
\text { STF }\end{array}$ & $\begin{array}{c}\text { HE- } \\
\text { LTF1 }\end{array}$ & $\cdots$ & $\begin{array}{c}\text { HE- } \\
\text { LTFN }\end{array}$ & $\begin{array}{c}\text { HE } \\
\text { Data }\end{array}$ \\
\hline
\end{tabular}

b

\begin{tabular}{|c|c|c|c|c|c|c|c|c|c|c|c|c|}
\hline L-STF & \multirow{2}{*}{ L-LTF } & L-SIG & RL-SIG & $\begin{array}{c}\text { HE- } \\
\text { SIG-A }\end{array}$ & $\begin{array}{c}\text { SA- } \\
\text { SIG-A }\end{array}$ & $\begin{array}{c}\text { HE- } \\
\text { SIG-B }\end{array}$ & $\begin{array}{c}\text { SA- } \\
\text { SIG-B }\end{array}$ & $\begin{array}{c}\text { HE- } \\
\text { STF }\end{array}$ & $\begin{array}{c}\text { HE- } \\
\text { LTF1 }\end{array}$ & $\ldots$ & $\begin{array}{c}\text { HE- } \\
\text { LTFN }\end{array}$ & $\begin{array}{c}\text { HE } \\
\text { Data }\end{array}$ \\
\hline
\end{tabular}

Fig. 5 Frame format: a HE MU PPDU format in IEEE 802.11 ax and $\mathbf{b}$ the proposed SA-HE mode 
defined to have a common field followed by a user-specific field. The common field includes the information for all of the designated users to receive the PPDU in their corresponding bandwidth. The user-specific field consists of the packet size, modulation scheme, and coding level for the packet of the specified user among all simultaneously selected users. The HE-STF field is used to improve AGC performance at the receiver in a MIMO setting, and the HE-LTF is used to estimate MIMO channels. The data field contains the modulated signals, which overlap in both time and spectrum across all simultaneous users [1]. The HE-SIG-A embodiment is composed of two symbols, HE-SIG-A1 and HE-SIG-A2 (as depicted in Fig. 6), which are currently proposed in the IEEE 802.11ax amendments $[2,30]$. All the information shown in Fig. 6 is required for the receiver to interpret the HE transmission configuration. Specifically, group ID is used to specify the user information for each transmission. First, each authorized user is assigned a group ID and a user position by the AP via a group ID management frame. Each user served by the AP has a unique group ID and a corresponding user position. When a user receives a HE-SIG-A packet and finds that the group ID in that packet matches its own local group ID, the user will look up the user position and then choose the modulation parameters that belong to that user position in order to decode the received signal. The HE-SIG-A field is designed for MU-MIMO operation. However, in our selectivity-aware multi-user scheme, since we divide the entire wireless channel into multiple sub-channels, the signal of a user may occupy different number of sub-channels depending on the channel characteristics of the simultaneous users. For a selected user, to correctly decode the signals, the index of every sub-channel that carries its data packets must be known. Moreover, for different sub-channels, the resulting signal-to-interference-plus-noise ratio (SINR) is different. As a result, different modulation and coding schemes (MCSs) may be applied to different sub-channels, even for the same user. Hence, MCS information must also be conveyed to each intended receiver, and we correspondingly insert additional signaling fields in the frame to carry this information. We use 3 of the reserved bits (shown in Fig. 6) in the HE-SIG-A fields to inform the receiver whether the transmission is in selectivity-aware mode or not and how many sub-channels are in use. The configuration of the bandwidth division is shown in Table 1. The number of coded bits in the base rate transmission mode is given by $N_{\text {base, }}$, the number of resulting sub-channels is denoted as $N_{\text {sub }}$, and the number of additional SA-SIG-A and SA-SIG-B symbols is $N_{a}$ and $N_{b}$, respectively. For each bandwidth, a corresponding number of sub-carriers are used in the OFDM scheme. $N_{\text {pkt-bit }}$ is the number of bits used to indicate the packet size on each sub-channel for each user. When the AP uses the existing IEEE 802.11ax scheme for transmission, the selectivity-aware configuration is set to the value $v=" 000 "$. A non-zero selectivity-aware configuration denotes that the AP will divide the channel into $2^{v}$ sub-channels for transmission. In this mode, there are additional selectivity-aware signal-A (SA-SIG-A) symbols appended after the HE-SIG-A field and SA-SIG-B symbols appended after the HE-SIG-B field to inform the receiver the configurations on each sub-channel. The frame format of the SA-HE mode is shown in Fig. 5b.

For each sub-channel, a separate group ID, NSTS (number of space-time streams), coding type, MCS value, and packet size are needed for the receivers to correctly decode the packet. The group ID, NSTS, and coding type are carried in the HE-SIG-A and SA-SIG-A fields. The MCS value and packet size are carried in the HE-SIG-B field and SA-SIG-B fields. With sub-channel divisions, the HE-SIG-A field contains the group ID, NSTS, and coding type for the first sub-channel. The group ID, NSTS, and coding type for the remaining sub-channels are in the SA-SIG-A field. Each group ID has 6 bits, each NSTS has 3 bits, and each coding type has 1 bit. For each sub-channel, the maximum simultaneous users is 4 . As a result, for each sub-channel, 22 additional bits need to be transmitted

a

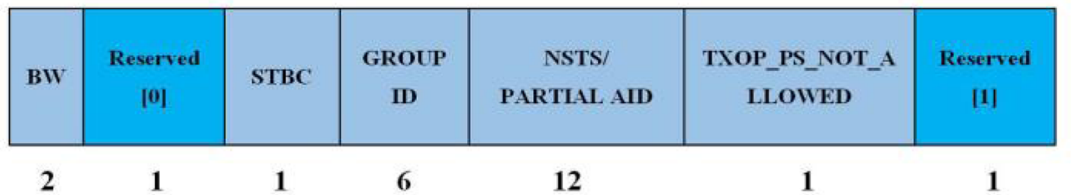

Bits:

2

b

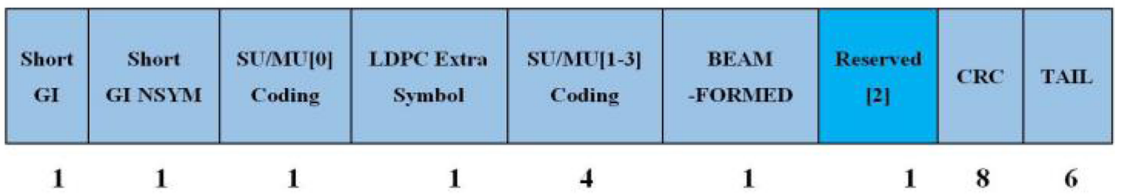

Fig. 6 Proposed HE-SIG field in IEEE 802.11ax: a HE-SIG-A1 and b HE-SIG-A2 
Table 1 Selectivity-aware configuration

\begin{tabular}{|c|c|c|c|c|c|c|}
\hline Bandwidth & $N_{\text {base }}$ & $v$ & $N_{\text {sub }}$ & $N_{\text {pkt-bit }}$ & $N_{a}$ & $N_{b}$ \\
\hline \multirow[t]{6}{*}{$20 \mathrm{MHz}$} & 33 & 000 & 1 & 16 & 0 & 0 \\
\hline & & 001 & 2 & 15 & 2 & 1 \\
\hline & & 010 & 4 & 14 & 3 & 2 \\
\hline & & 011 & 8 & 13 & 7 & 4 \\
\hline & & 100 & 16 & 12 & 14 & 8 \\
\hline & & 101 & 32 & 11 & 29 & 15 \\
\hline \multirow[t]{6}{*}{$40 \mathrm{MHz}$} & 65 & 000 & 1 & 17 & 0 & 0 \\
\hline & & 001 & 2 & 16 & 2 & 1 \\
\hline & & 010 & 4 & 15 & 3 & 1 \\
\hline & & 011 & 8 & 14 & 7 & 3 \\
\hline & & 100 & 16 & 13 & 14 & 5 \\
\hline & & 101 & 32 & 12 & 29 & 8 \\
\hline \multirow[t]{6}{*}{$80 \mathrm{MHz}$} & 136 & 000 & 1 & 19 & 0 & 0 \\
\hline & & 001 & 2 & 18 & 2 & 1 \\
\hline & & 010 & 4 & 17 & 3 & 1 \\
\hline & & 011 & 8 & 16 & 7 & 2 \\
\hline & & 100 & 16 & 15 & 14 & 3 \\
\hline & & 101 & 32 & 14 & 29 & 5 \\
\hline \multirow[t]{6}{*}{$160 \mathrm{MHz}$} & 272 & 000 & 1 & 19 & 0 & 0 \\
\hline & & 001 & 2 & 18 & 2 & 1 \\
\hline & & 010 & 4 & 17 & 3 & 1 \\
\hline & & 011 & 8 & 16 & 7 & 1 \\
\hline & & 100 & 16 & 15 & 14 & 2 \\
\hline & & 101 & 32 & 14 & 29 & 3 \\
\hline
\end{tabular}

with the base rate in the SA-SIG-A field. We use a different number of packet-size configuration bits for each sub-channel according to each sub-channel division to make the maximum physical layer frame duration backward compatible. The packet size and MCS information for the first sub-channel is located in the HE-SIG-B field, and the packet size and MCS information for the rest of the sub-channel are in the SA-SIG-B field. The number of SA-SIG-A and SA-SIG-B symbols for each channel bandwidth and channel division is shown in Table 1. Since the SA-SIG-A field uses the IEEE $802.11 \mathrm{a} / \mathrm{g}$ legacy base rate (24 bits per symbol) for the purpose of backward compatibility, the number of symbols in the SA-SIG-A field, $N_{a}$, can be calculated as follows:

$$
N_{a}= \begin{cases}0 & \text { if } N_{\mathrm{sub}}=1 \\ \left\lceil\frac{\left(N_{\mathrm{sub}}-1\right) \times 22+6}{24}\right\rceil & \text { otherwise }\end{cases}
$$

In IEEE 802.11ax, each OFDM symbol with the base rate mode (BPSK with a code rate of $1 / 2$ ), 33, 65, 136, and 272 coded bits can be carried with the bandwidth of 20 , 40, 80, and $160 \mathrm{MHz}$, respectively [1]. The number of bits required to convey MCS information is 4 . As a result, the number of SA-SIG-B field symbols, $N_{b}$, can be calculated as follows:

$$
N_{b}= \begin{cases}0 & \text { if } N_{\text {sub }}=1 \\ \left\lceil\frac{\left(N_{\text {sub }}-1\right) \times\left(N_{\text {pkt-bit }}+4\right)+6}{N_{\text {base }}}\right\rceil & \text { otherwise }\end{cases}
$$

Compared to the current IEEE 802.11ax, the SA-SIG-A and SA-SIG-B fields introduce different overhead levels proportional to the channel bandwidth and channel division level. The throughput efficiency with overhead is given by:

$$
\xi=\frac{T_{f}-T_{\text {header }}-\left(N_{a}+N_{b}\right) \times T_{s}}{T_{f}-T_{\text {header }}}
$$

Here, $T_{f}$ is the PHY frame duration. $T_{\text {header }}$ is the time duration, including the preambles and signaling symbols in the frame. $T_{s}$ is a symbol duration with a value of $13.6 \mu \mathrm{s}$, assuming a cyclic prefix (CP) length of $0.8 \mu \mathrm{s}$ as specified in [1]. From the evaluation in the following section, we will see that the improvement provided by channel division is much higher than the overhead introduced by channel division.

\subsection{Physical layer modulation}

For the SA-HE scheme, since we divide the entire channel into sub-channels with the same frame duration, the packet size transmitted on one sub-channel is smaller than the existing IEEE 802.11ax HE mode. The AP first divides the packet for a served user into smaller packets that will fit into one sub-channel. Then, the AP allocates one or more small packets for a user on one or more subchannels with potentially different modulation and coding schemes. The AP first calculates the MCS for each user on each sub-channel according to the channel quality and orthogonality. For each sub-channel, the AP allocates a certain amount of information bits to make sure each subchannel has the same number of valid data symbols. The PHY layer transmitter block diagram of the SA-HE mode is shown in Fig. 7a. Currently, an IEEE 802.11ax AP modulates the data for each served user and then maps the data onto the analog and RF chains. In the proposed SAHE scheme, there is an additional sub-channel dimension. The AP needs to modulate the data for each user on each sub-channel and then maps the resulted data into spatial and sub-channel streams. While SA-HE has a slightly higher complexity, modern wireless transceivers typically use a combination of an embedded CPU and ASIC which can both be multiplexed over time.

\subsection{Media access control layer design}

The MAC layer of the SA-HE mode mostly adopts the MAC protocol for the HE mode of the latest draft of IEEE 802.11ax with some modifications [2]. Since the entire channel is divided into multiple sub-channels, and a separate subset of users is served on each sub-channel, one AP may support a larger number of simultaneous users than 


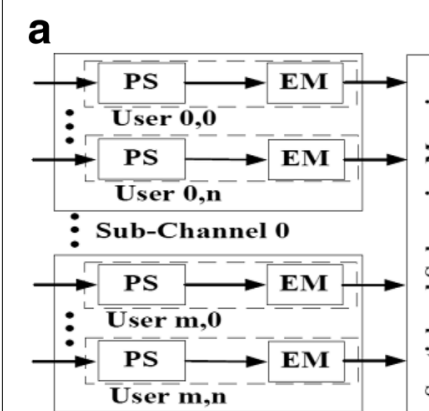

Sub-Channel m

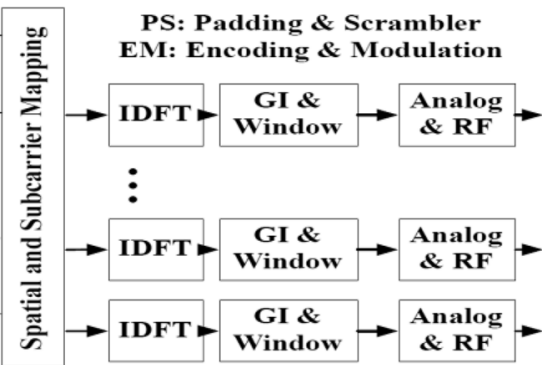

Window $\rightarrow \begin{gathered}\text { Analo } \\ \text { \& RF }\end{gathered}$ b

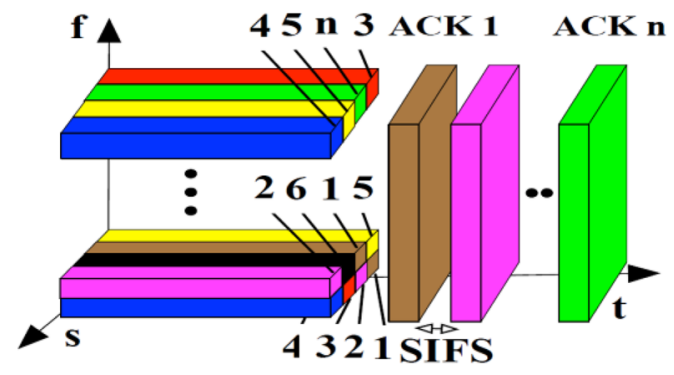

Fig. 7 SAMU MAC and PHY design: a transmitter block diagram of SA-HE mode and $\mathbf{b}$ transmission and acknowledgement in SA-HE. There are $n$ users, and at each sub-channel, four users are grouped together. The set of users at different sub-channels may or may not overlap

the current number supported by IEEE 802.11 APs. In this section, we describe how SA-HE manages and supports the larger number of simultaneous users.

According to the reported channel characteristics from all the authorized users via sounding packets, the AP selects the optimal subset of users for each sub-channel. Priority from the application layers or fairness could also be considered during user selection. However, in our design and experimentation, we choose to use the maximum total throughput metric to select the users. The user selection mechanism is based on a low-complexity zeroforcing beamforming strategy that chooses the candidate channel with the best orthogonality [29]. The process is depicted in Algorithm 1.

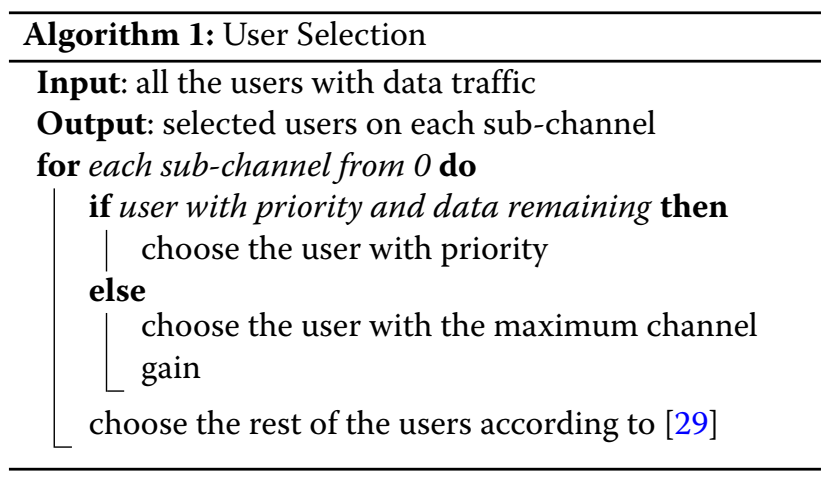

Adopted from IEEE 802.11ax, each user is assigned a distinct user group ID and user position via the group ID management frame. As we described in the PHY layer design, there is information about the group ID and user position contained in the HE-SIG-A and SA-SIG-A fields. Whenever the AP transmits packets to multiple users on multiple sub-channels, each user receives the HE-SIG-A and SA-SIG-A fields in their packets. With the information contained in the signaling symbols, the user knows the information of all the other simultaneous users, as well as the user position information for each sub-channel.
The receiver then looks up the stored user group table and generates a list indicating the simultaneous users for this transmission. The users served with this transmission are then ordered from lower sub-channel to higher subchannel and from lower position to higher position. The users of sub-channel 0 is at the front of the list, and the user with user position 0 on sub-channel 0 is the first item in the list. Then, the receiver checks the next sub-channel. If the users on the current sub-channel already exist in the list, then they will not be included again. If there are new users that do not exist in the list, the receiver will append the new users to the list. The receiver repeats this process until the last sub-channel is considered, as shown in Algorithm 2.

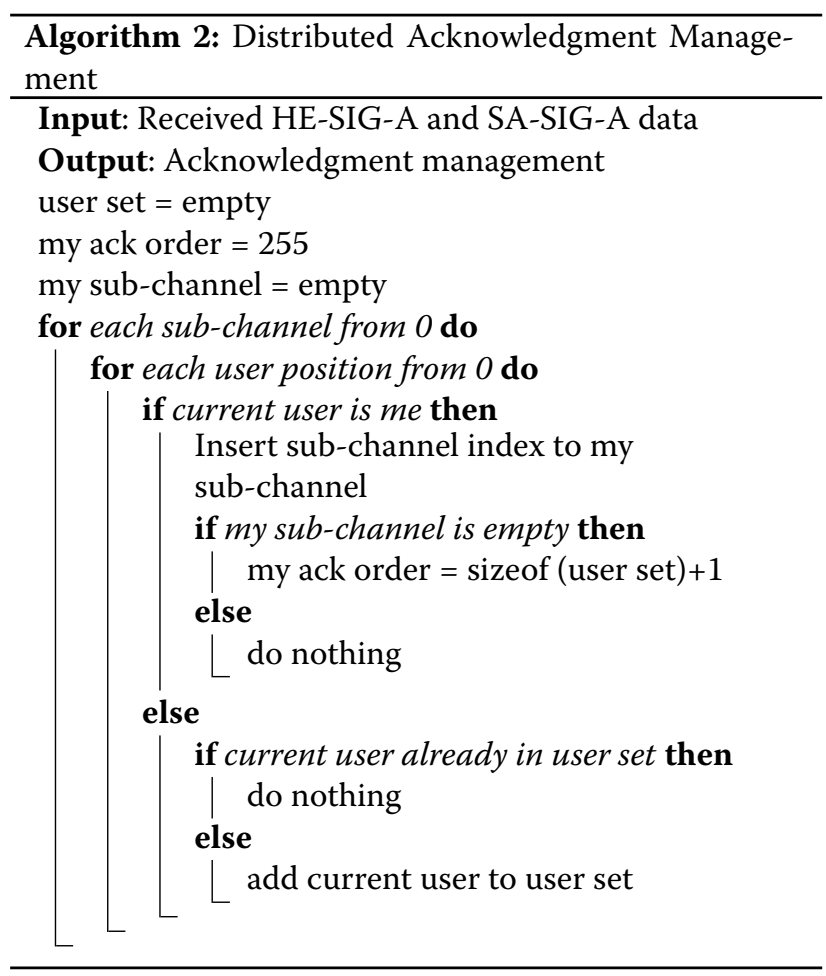


In Fig. 7b, we depict an example explaining the ordering mechanism. On sub-channel 0 , the group of users $\{1,2,3,4\}$ are served. On sub-channel 1 , the group of users $\{5,1,6,2\}$ are served. Then, for user 2 , two lists are created. The first list is the user order list, which is $\{1,2,3,4,5,6\}$. The second list is the sub-channel list that carries packets for user 2 . In this case, it is sub-channel 0 , sub-channel 1 \}.

For each user, after decoding its PHY layer packets on one or more sub-channels according to the sub-channel list, it will recover the MAC layer packets by combining the PHY layer packets. When the receiver successfully receives the data packets on all sub-channels, an ACK packet is required to be sent back to the AP. Otherwise, the AP will assume packet loss and a packet retransmission would be initiated in the following transmission. With the user order list, the users that have successful packet receptions will send ACK packets one by one to notify the AP. The ACK packets include the user and sub-channel information, according to the order in the list. Since the ACK transmission time can be pre-calculated and followed by a DIFS slot, other users in the queue can virtually sense the total number of ACK frames.

Sub-channel division will reduce the capacity of each sub-channel proportionally to the number of subchannels. However, each user can occupy more than one sub-channel. By allocating the same user multiple subchannels, SAMU can meet the data rate demands of users, while achieving significant gains in the aggregated throughput among all users. During user selection, the AP can mark some users as having higher priority. For each sub-channel, the user with the highest priority and highest channel gain will be selected first. Then, the users with the best channel orthogonality will be selected accordingly.

The ACK packets from multiple users usually cause additional transmission overhead. However, this overhead can be reduced by two means. First, SAMU users send ACK packets back-to-back according to the user order in the PHY header, which removes the overhead of block ACK request packets in IEEE 802.11ax specifications $[1,2]$. Second, by partially overlapping the user sets for different channels, the total number of users in one transmission can be minimized. Thus, the number of back-to-back ACK packets can be minimized.

\subsection{Channel division adaptation}

Due to the mobility of the transmitter, receiver, or obstacles within the environment, channel fading is observed in the time domain, including variations of the number of taps, the amplitude of each tap, and the tap delays.

In the frequency domain, the time-varying wireless channel is represented by amplitude and phase shifts on each sub-carrier and the coherence bandwidth of the channel spectrum. Coherence bandwidth is an approximate maximum bandwidth or frequency interval over which two frequencies of a signal are likely to experience comparable or correlated amplitude fading. When the coherence bandwidth is small, the signal will experience significantly different channel responses at different frequencies across a wideband channel. Therefore, to maintain orthogonality between users, we should divide the entire bandwidth into more sub-channels. When the coherence bandwidth is large, we can divide the whole bandwidth into less sub-channels to reduce the overhead introduced by the system information carrying the channel division information.

If we divide the entire channel into a small number of sub-channels, then each channel may still experience severe frequency selectivity, not being able to provide the optimal performance improvement. If we divide the entire channel into a large number of sub-channels, the channel orthogonality will become much better, potentially increasing the system throughput. However, the overhead caused by the control information will also degrade the system performance. In order to optimize the system, we need to divide the channel into different number of subchannels, according to the level of frequency selectivity. Delay spread is one of the key parameters that determines the frequency selectivity. The channel characteristics on each sub-channel typically change with time or due to diversity across environments. As a result, the subset of users on each sub-channel should also be updated to adapt to the channel conditions.

Different levels of channel selectivity require unique channel division factors to achieve the optimal performance, which is a balance between the throughput gain introduced by the channel division and the overhead for conveying the division information. The AP measures the RMS delay spread of channels for all the authorized users periodically and then changes the band division factors accordingly. The number of resulting sub-channels from channel division, $m$, can be expressed as:

$$
m=2^{\left\lfloor\log _{2}\left(W \tau f\left(T_{f}\right)\right)\right\rceil}
$$

Here, $L\rceil$ represents a rounding function, and $f\left(T_{f}\right)$ is a function of the frame duration. Even for the same channel characteristics, different frame durations result in different number of sub-channels due to the different overhead caused by the different channel division settings. Since it is challenging to find a closed form expression for $f\left(T_{f}\right)$, we choose to empirically find a set of delay-spread thresholds between different options of the sub-channel divisions, which is shown in Section 6. With environments that do not change significantly with time, the AP may always keep the same number of sub-channels for transmission. However, once the environment changes, causing the measured delay spread to cross a threshold, the AP will adapt the number of sub-channels accordingly. 
The frequency-domain MIMO channel response is usually estimated by the received HE-LTF signals and the local HE-LTF signals. The receiver then uses the estimated channel response to remove the distortions of received symbols for decoding. However, for our delay-spread measurements, it is more convenient to process the timedomain channel profile. Therefore, we apply an inverse fast Fourier transform (IFFT) to the estimated frequency domain channel response to generate the time-domain channel profile, demonstrated in Fig. 8.

\section{Experimental setup}

In order to evaluate SAMU on diverse channels, we use a software-defined radio architecture, WARPLab, in both emulated channels and a representative indoor environment, as shown in Fig. 9. WARPLab enables users to implement the PHY layer and MAC layer functionalities in MATLAB and transmit/receive actual signals using RF radios [26]. Then, the coded and modulated data samples are transferred to the WARP board via an Ethernet cable. WARP is then triggered to transmit data samples over the air. The receiver samples the received signal over the air and then transfers the raw samples to the PC, where MATLAB demodulates and decodes the transmitted data. In our experiments, one WARP board with four antennas acts as an AP, and the rest of the WARP boards act as client devices equipped with one antenna for each. We use an Azimuth Wireless Channel Emulator to investigate the factors that affect the system performance. The channel emulator can generate controllable and repeatable channel conditions for complex wireless environments by ways of a documented TCL API for scripting tests. The emulation system is shown in Fig. 9a.

In addition to the emulator experiments, we also evaluate the system in an indoor environment. The floor plan of the office environment for our experiments is shown in Fig. 10. We place the AP at the location marked with the red star and randomly place the users in the area marked with green ovals. The antennas of the AP form a square configuration shown in Fig. 9b. The distance between antennas along each side of the square is $20 \mathrm{~cm}$, which is approximately the size of an AP device. Moreover, with the application of the 2.4 and $5.2 \mathrm{GHz}$ bands, this antenna separation allows little correlation between the channels from different transmit antennas.

We run our experiments on a WiFi channel at two central frequencies: 2.4 and $5.2 \mathrm{GHz}$. The transmit signal bandwidth is $20 \mathrm{MHz}$, which is the maximum bandwidth currently supported by WARP. We use 256 OFDM sub-carriers in our experiments, which is compatible for next-generation WLAN technologies with a $20 \mathrm{MHz}$ bandwidth.

For wireless systems, throughput is a typical metric of system performance. However, the throughput is usually closely related to the modulation and coding scheme (MCS). For a system with sparse MCS choices, in two

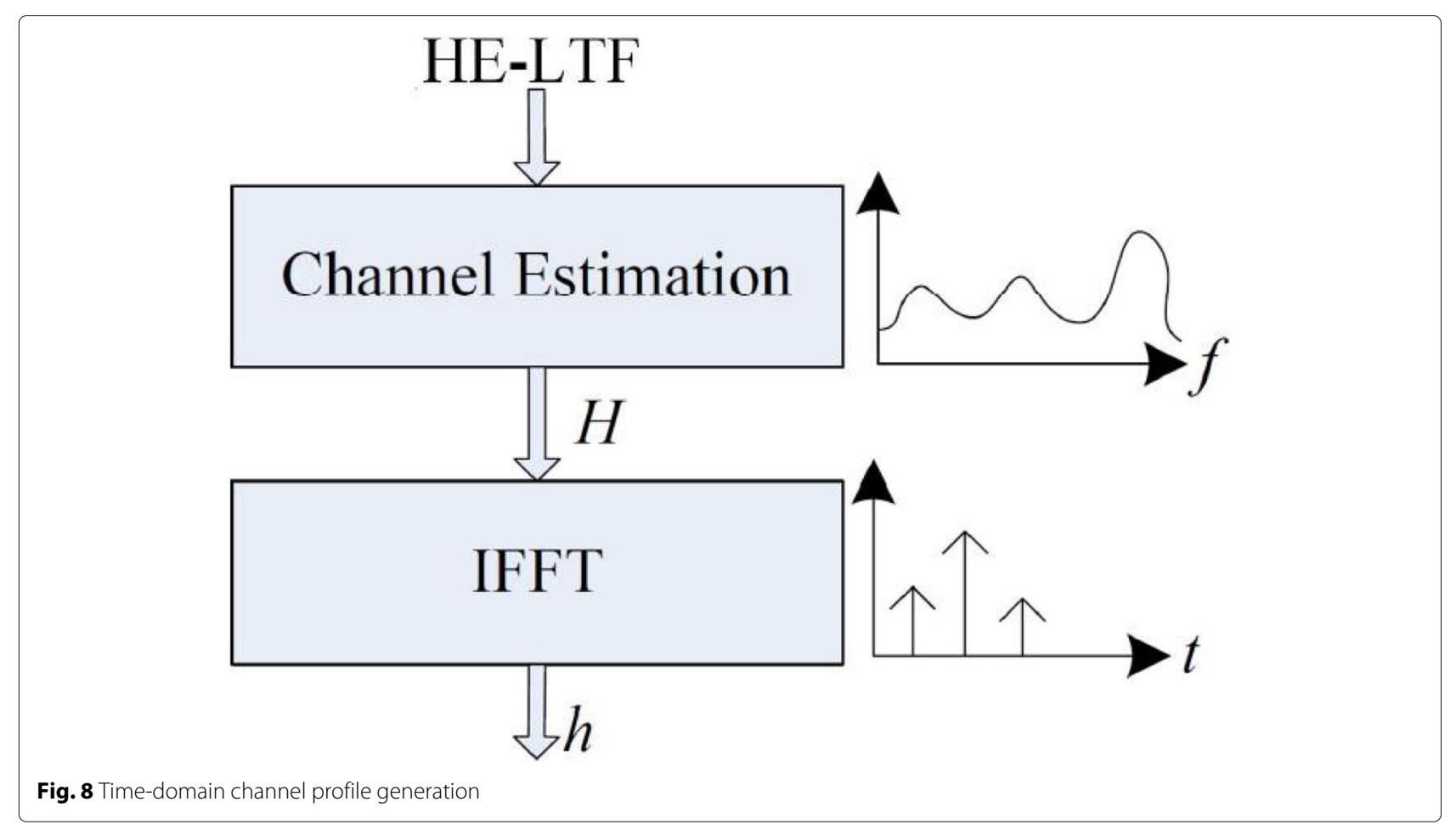



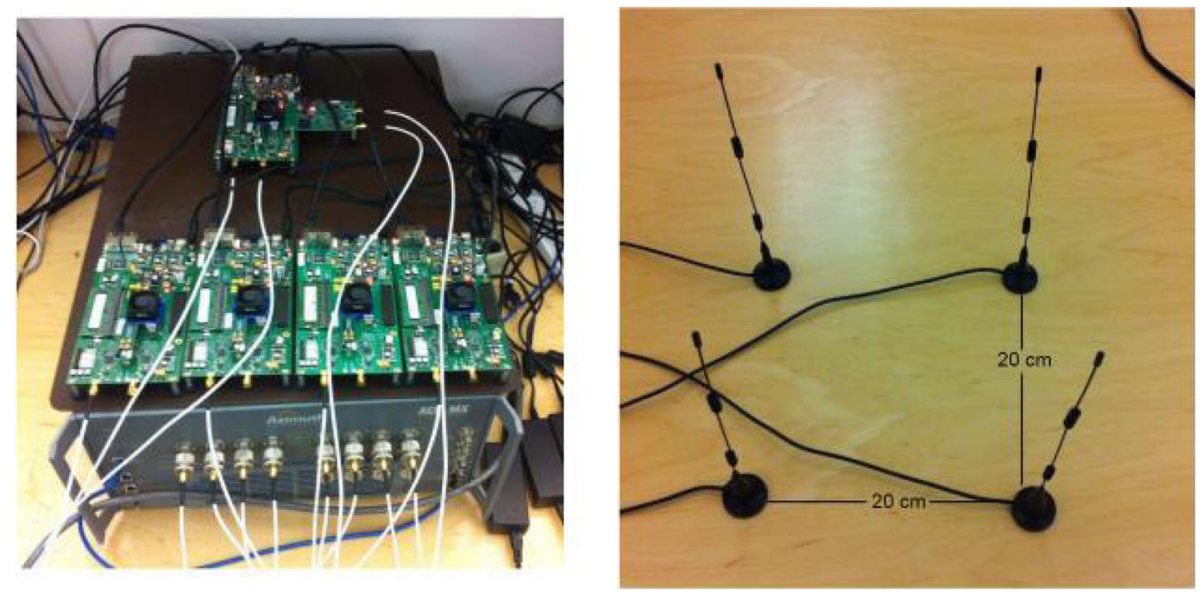

Fig. 9 Equipment settings for performance evaluation. a Setup using WARP and Azimuth Channel Emulator. b Antenna placement for indoor over-the-air testing

scenarios with different channel qualities, the same MCS may be selected. As a result, the same throughput will be reported to the higher layers. Moreover, rate adaptation is usually used for wireless systems to adapt the transmission rate to the channel quality to improve the system throughput. However, due to the sparse MCS options and the performance of various rate adaptation schemes, the reported throughput can be diverse even for the same physical system design, preventing direct isolation of the potential system performance gain introduced by our selectivity-aware MU-MIMO scheme. Therefore, we adopt the system evaluation metric and method used in [8], which is to measure the received SNR and directly map the SNR to throughput. The SNR is calculated by mapping the digital output samples to the absolute received signal power applied by amplifier gains.
Assuming $i$ is the sub-channel index, the throughput metric is given by:

$$
\text { Rate }=\sum_{i} \log _{2}\left(1+\mathrm{SNR}_{\text {measured }}^{i}\right)[\mathrm{bit} / \mathrm{s} / \mathrm{Hz}]
$$

\section{Results and discussion}

In this section, we discuss the channel division across different values of and evaluate the performance gain of SAMU in two types of environments: emulated channels and practical channels, as introduced in the previous section.

\subsection{Channel division across delay spreads}

We first use emulated channels to study the impact of different channels and system parameters. For each

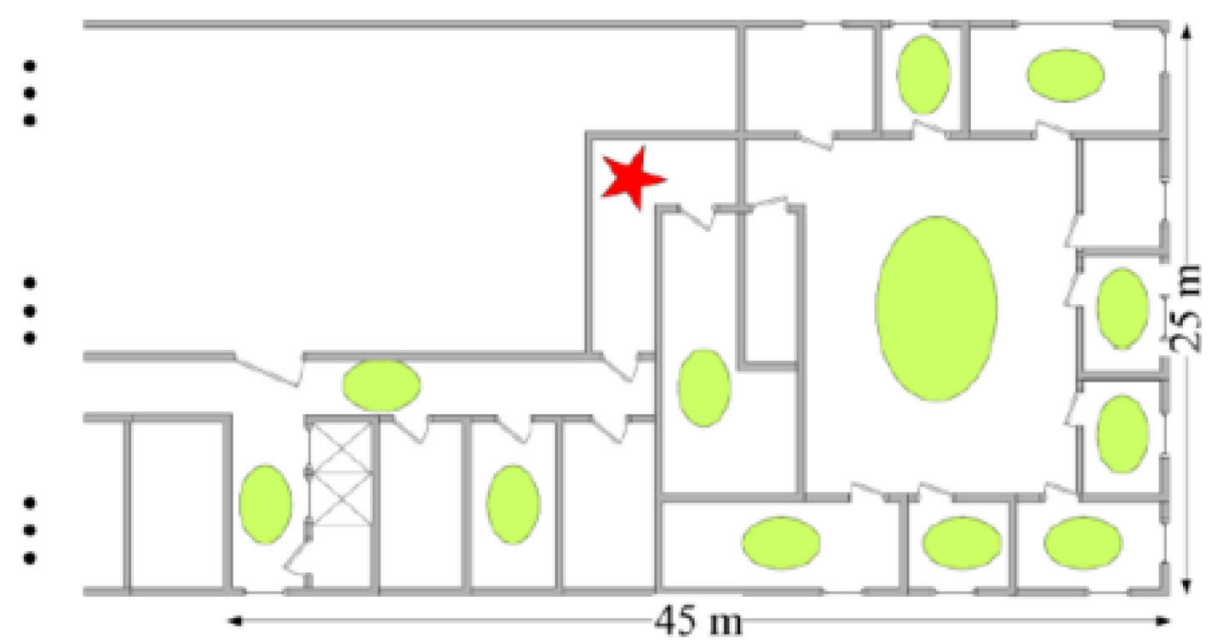

Fig. 10 Floor plan of the indoor testing environment 
sub-channel division factor, we evaluate the throughput improvement versus the delay spread to find the optimal division factor given a particular delay spread.

We first study the impact of diverse delay spreads on channel divisions. We set the total number of users served by an AP to be 256 and use an equal-power echo channel in this experiment (i.e., there is no attenuation in gain from the first channel tap to the second). We set the number of equal-power channel taps from 1 to 7 and the time delay between adjacent channel taps to be $50 \mathrm{~ns}$, which is the inverse of the channel bandwidth. The total channel gain for different channel models is normalized to $0 \mathrm{~dB}$. For each test, we generate multi-path channel effects between each of the transmit-receive antenna pairs with a Rayleigh distribution. Then, we measure the throughput with diverse delay spread values for each channel division configuration listed in Table 1. For each test case, we complete 1024 transmissions to obtain a reliable average result, with a packet over-the-air duration of $2.73 \mathrm{~ms}$ and a guard interval of $3.2 \mu \mathrm{s}$. The results are given in Figs. 11a and 12a.

We can observe that, without channel division (i.e., one sub-channel), a non-zero delay spread will degrade the system throughput severely. With channel divisions, less frequency selectivity occurs within each sub-channel, achieving far better channel orthogonality than that without channel divisions. One should not ignore that the channel division increases the throughput only logarithmically with respect to the number of sub-channels. By further increasing the number of sub-channels (e.g., more than 16), we predict that the throughput performance of SAMU is about to significantly increase. Moreover, for a fixed bandwidth (e.g., $20 \mathrm{MHz}$, as in our experiments), the performance improvement of SAMU is less for a smaller delay spread due to less frequency selectivity and less of a need to divide into sub-channels. However, as bandwidth scales (e.g., $160 \mathrm{MHz}$, as in 802.11ac and 802.11ax), even a very small delay spread (less than $50 \mathrm{~ns}$ ) can result in severe frequency selectivity across the bandwidth. We expect that the performance of SAMU will only increase from the results reported in this paper as instantaneous bandwidth scales.

To quantify the gain, we show the improvement introduced by channel division in Figs. $11 \mathrm{~b}$ and $12 \mathrm{~b}$. We observe that, for 4 antennas and 4 selected users on each sub-channel, when the delay spread reaches $300 \mathrm{~ns}$, we can achieve as much as 75.4 and $84.8 \%$ improvement compared to the current IEEE 802.11ax MU-MIMO scheme without channel divisions for 2.4 and $5.2 \mathrm{GHz}$, respectively. At both frequency bands, the channel division has less of an improvement with a delay spread of $50 \mathrm{~ns}$ because of the reduced frequency selectivity with a $20-\mathrm{MHz}$ bandwidth. However, with higher bandwidth, the trends observed here will collectively shift to the left (i.e., the effect of frequency selectivity is exacerbated even with small delay spreads, and the use of sub-channels will only have a greater benefit for a given delay spread). Moreover, the 2.4-GHz band has benefited from higher data

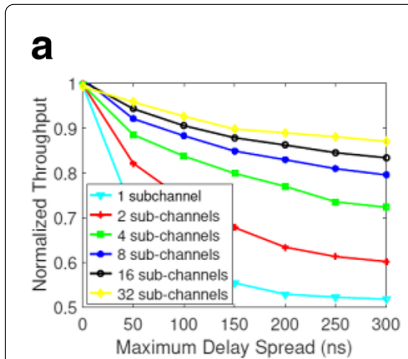

e

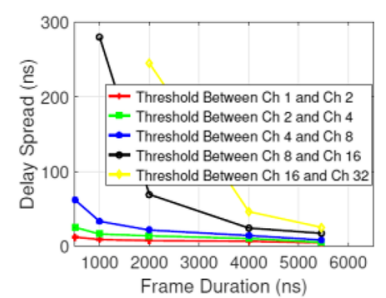

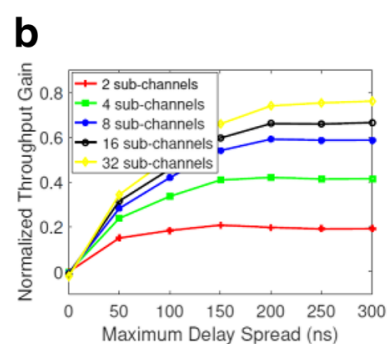

f

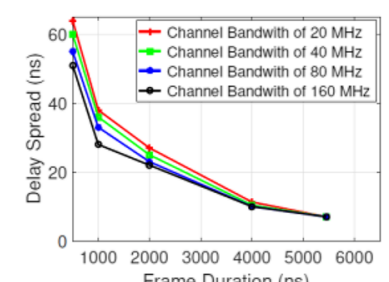

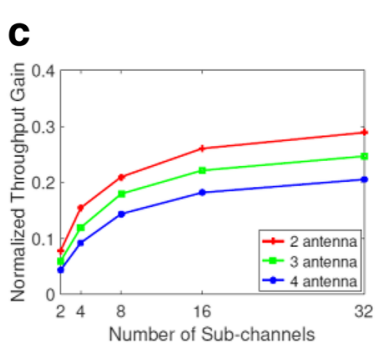

g

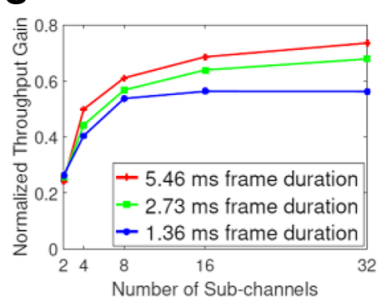

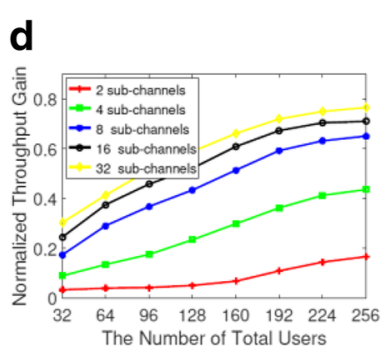

h

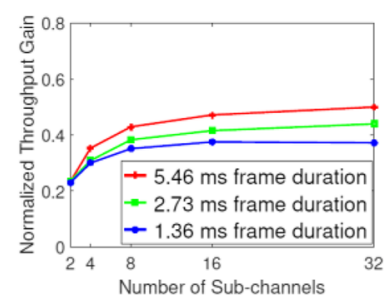

Fig. 11 Experimental results with either emulated or actual wireless channels on $2.4 \mathrm{GHz}$. a Normalized throughput for different number of sub-channels across channel delay spreads. b Normalized throughput gain for different number of sub-channels across channel delay spreads. c Normalized throughput gain versus antenna numbers on the AP for 2 selected users. d Throughput gain versus the number of total users in the coverage of the AP. e Sub-channel division threshold changes with frame duration. f Sub-channel division threshold changes with channel bandwidth. $\mathbf{g}$ Normalized throughput gain in emulated IEEE TGn channel model. h Normalized throughput gain in an actual office environment 


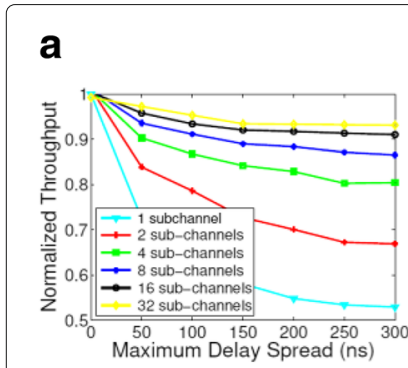

re

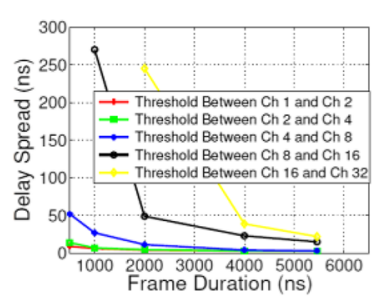

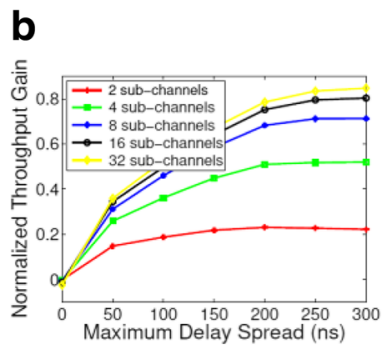

$f$

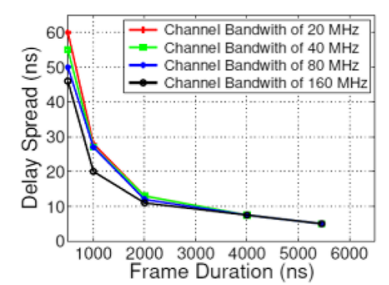

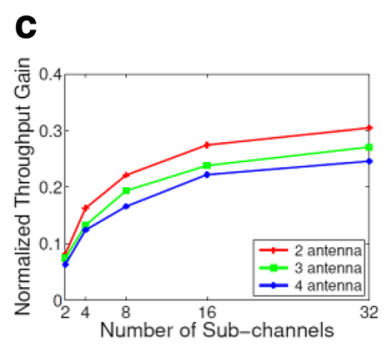
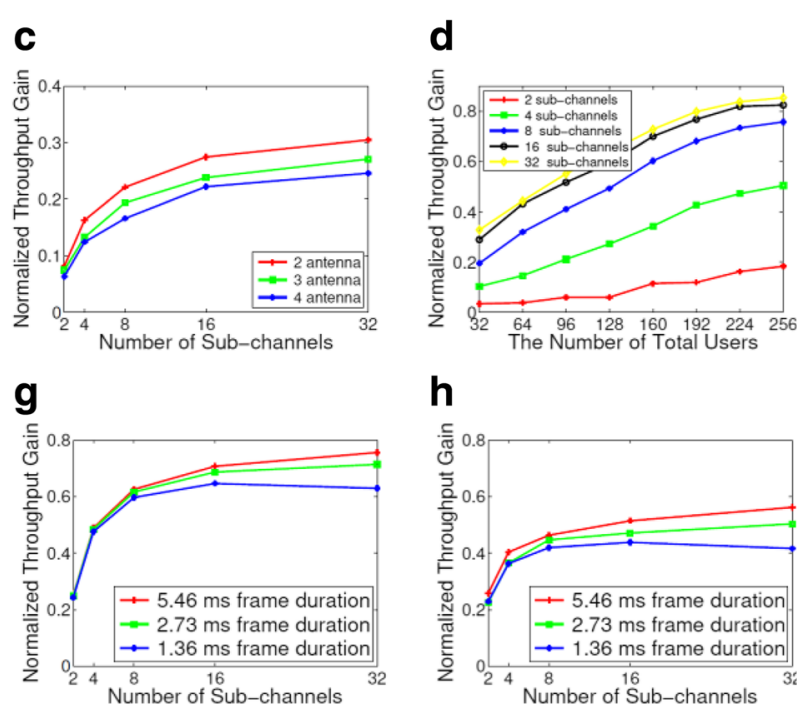

h

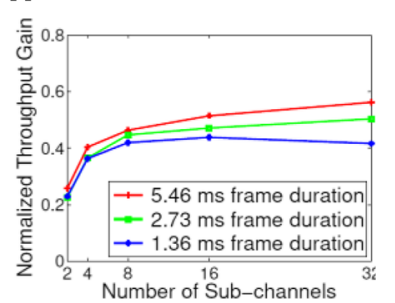

Fig. 12 Experimental results with either emulated or actual wireless channels on $5.2 \mathrm{GHz}$. a Normalized throughput for different number of sub-channels across channel delay spreads. b Normalized throughput gain for different number of sub-channels across channel delay spreads. c Normalized throughput gain versus antenna numbers on the AP for 2 selected users. $\mathbf{d}$ Throughput gain versus the number of total users in the coverage of the AP. e Sub-channel division threshold changes with frame duration. f Sub-channel division threshold changes with channel bandwidth. $\mathbf{g}$ Normalized throughput gain in emulated IEEE TGn channel model. h Normalized throughput gain in an actual office environment

rates compared to the $5.2 \mathrm{GHz}$ under the same delay spread.

We also examine how the throughput is affected by the number of antennas on the AP. To do so, we keep the number of selected users at 2 and change the number of AP antennas to be 2,3 , and 4 . We use a 7-tap, equal-power echo channel model for this experiment. The result is shown in Figs. 11c and 12c. We observe that, as the number of antennas increases on the AP, the achieved channel division improvement decreases for both frequency bands. This is due to channels between users tending to be more orthogonal, regardless of the frequency selectivity, as the number of antennas become much more than the number of selected users. In addition, the throughput performance improvement due to channel division is more significant in the $5.2-\mathrm{GHz}$ band as compared with the 2.4-GHz band.

As the number of users increases, the probability of finding completely orthogonal simultaneous users increases. Hence, dividing the bands according to the level of frequency selectivity could achieve far greater throughput gains (i.e., where orthogonality in space cannot be exploited, orthogonality in frequency can be exploited). To evaluate this experimentally, we return to the default setup of 4 antennas on the AP and again use a 7-tap equalpower echo channel model. The performance for a different number of total users (with at most 4 users selected per transmission) is shown in Figs. $11 \mathrm{~d}$ and $12 \mathrm{~d}$. We observe that, the throughput improvement increases with the increase of the number of users for both frequency bands. However, the increase becomes more nominal when the total user number exceeds approximately 160 users and 192 users for 2.4 and $5.2 \mathrm{GHz}$, respectively.

Furthermore, SAMU is able to adapt the number of sub-channels to the delay spread and frame duration. We now evaluate the adaptation threshold for different frame durations within emulated channels and show the results in Figs. 11e and 12e. We observe that as the frame duration increases, the sub-channel adaptation threshold decreases, tending to use more sub-channels for the transmission. This is due to the reduction in relative overhead for the channel division with a larger frame duration, as indicated in Eq. 6. We also show that $2.4 \mathrm{GHz}$ has a larger appetite for more sub-channel divisions than $5 \mathrm{GHz}$, due to the larger multi-path effect from which it suffers. Similarly, with greater bandwidth, the SA-SIG-B field will have less symbols, reducing the impact of the channel division overhead. Therefore, the adaptation threshold also decreases, as shown in Figs. $11 \mathrm{f}$ and $12 \mathrm{f}$.

\subsection{Diverse 802.11 channels}

For our system evaluation, we first use a channel emulator with channel model $\mathrm{E}$ in IEEE 802.11 Wireless LANs TGn Models, which is for typical large indoor spaces with NLOS conditions (e.g., a large auditorium, conference hall, or gymnasium) [31]. The large number of users in these locations tend to improve MU-MIMO performance. The channel model profile we use is shown in Table 2. We use a central frequency of $5.2 \mathrm{GHz}$. We vary the frame duration to investigate the impact of the 
Table 2802.11 indoor channel model

\begin{tabular}{llllll}
\hline Excess delay (ns) & 0 & 10 & 20 & 30 & 50 \\
Power (dB) & -2.6 & -3.0 & -3.5 & -3.9 & -4.5 \\
Excess delay (ns) & 80 & 110 & 140 & 180 & 230 \\
Power (dB) & -5.6 & -6.9 & -8.2 & -9.8 & -11.7 \\
Excess delay (ns) & 280 & 330 & 380 & 430 & 490 \\
Power (dB) & -13.9 & -16.1 & -18.3 & -20.5 & -22.9 \\
\hline
\end{tabular}

overhead introduced by the extra SA-SIG-A and SA-SIGB symbols. Moreover, we implement an OFDMA-based protocol for the legacy IEEE 802.11ax as a comparison to the performance of SAMU, independently. In order to better compare the system performance between SAMU and the legacy 802.11ax, the key evaluation feature is whether the frequency selectivity-awareness is implemented across the various emulated channels. Table 3 summarizes parameters of the IEEE 802.11ax standard and SAMU.

The results are shown in Figs. 11g and 12g. We observe that, with the largest frame duration setting, $5.46 \mathrm{~ms}$ specified in [1], SAMU achieves 70 and $75 \%$ throughput improvement over the standard HE mode in 802.11ax for 2.4 and $5.2 \mathrm{GHz}$, respectively. However, if the frame duration is smaller, the impact of the overhead introduced by the extra SA-SIG-A and SA-SIG-B symbols becomes larger, resulting in less improvement by channel division. In addition, when the number of divided sub-channels is 8 , the number of sub-carriers per sub-channel is comparable to the size of a 802.11ax Resource Unit (26 sub-carriers). Thus, we can predict that the size of a resource unit defined in legacy 802.11ax standard is not small enough to combat the frequency-selective fading, with excessive sub-channel division (smaller sub-carrier granularity) being able to further improve the system performance.

We also evaluated the performance gain of SAMU versus the legacy 802.11ax scheme without selectivityaware in the same practical office environment, as shown in Section 3. The legacy 802.11ax is based on the same OFDMA-based protocol for emulated channels. As depicted in Figs. 11h and 12h, SAMU outperforms the

Table 3 SAMU and IEEE 802.11ax OFDMA parameters

\begin{tabular}{lll}
\hline Protocol & IEEE $802.11 \mathrm{ax}$ & SAMU \\
\hline Central frequencies & $2.4 / 5.2 \mathrm{GHz}$ & $2.4 / 5.2 \mathrm{GHz}$ \\
FFT size & 256 & 256 \\
Modulation schemes & QPSK & QPSK \\
Coding rate & $\frac{1}{2}$ & $\frac{1}{2}$ \\
Guard interval & $3.2 \mu \mathrm{s}$ & $3.2 \mu \mathrm{s}$ \\
Selectivity-aware & No & Yes
\end{tabular}

existing 802.11ax scheme as much as 49 and $53 \%$ in an actual environment for 2.4 and $5.2 \mathrm{GHz}$, respectively. The reflections and multi-path in this office environment is not as severe as in the channel model that we use for channel emulation. If we conduct the experiments in an area with more reflections and larger delay spread, larger gains are expected. However, in an environment with smaller delay spread, as shown in Figs. $11 \mathrm{~h}$ and $12 \mathrm{~h}$, the $16 \mathrm{sub}-$ channel cases have better performance than the result with 32 sub-channels at the smallest frame duration of $1.36 \mathrm{~ms}$, suggesting that excessive sub-channel division can degrade the throughput due to the overhead introduced. While we still observe a dominant effect of large overhead when the number of sub-channels is greater than 16, our results show that the throughput gain of SAMU benefits from the increase of the number of sub-channels at relatively smaller overhead.

\section{Conclusion}

In this work, we considered frequency selectivity to optimize system capacity by jointly considering the critical enhancements of implementing OFDMA and MU-MIMO in the IEEE 802.11ax. We chose the optimal subset of users according to their spatial orthogonality and frequency diversity based on largest band division that can be considered frequency flat. SAMU ensures user optimality in each sub-channel, and we showed that it supports more simultaneous users and significantly improves the multi-user performance in a network. Moreover, we proposed a selectivity-aware high efficiency mode based on IEEE 802.11ax, which is an extension to the existing IEEE 802.11 standard. From our experimental results, SAMU showed as much as $84.8 \%$ throughput improvement compared to the existing systems in repeatable scenarios over the channel emulator and representative indoor environments. In the future, frequency selectivity will only increasingly dominate the behavior of WiFi as bandwidth scales, underscoring the importance of SAMU.

\section{Endnote}

${ }^{1}$ In this paper, the term selectivity refers to frequency selectivity.

\section{Abbreviations}

ACK: Acknowledgement; AGC: Automatic gain control; AP: Access point; API: Application programming interface; BSS: Basic service set; CP: Cyclic prefix; DIFS: Distributed coordination function interframe space; GI: Guard interval; IFFT: Inverse fast Fourier transform; LTF: Long training field; MAC: Media access control; MCS: Modulation and coding scheme; MU-MIMO: Multi-user multiinput multi-output; NDPA: Null data packet announcement; NLOS: Non-line of sight; NSTS: Number of spatial streams; OFDMA: Orthogonal frequency-division multiple access; PDF: Probability density function; PHY: Physical layer; PPDU: Physical protocol data unit; SA-HE: Selectivity-aware high efficiency; SDR: Software-defined radio; SINR: Signal-to-interference-plus-noise ratio; STBC: Space time block coding; STF: Short training field; TCL: Tool Command Language; TXOP: Transmission opportunity; WARP: Wireless open-access research platform; WiFi: Wireless fidelity; WLAN: Wireless local area network 


\section{Funding}

This work was in part supported by NSF grants: CNS-1318607, CNS-1320442, and CNS-C1526269.

\section{Availability of data and materials}

Not applicable.

\section{Authors' contributions}

YD conducted the detailed derivation of the proposed scheme. YD and YS carried out the most experiments and data analysis. YS improved the work and took charge of the paper submission. PC carried out the part experiments. EA, $\mathrm{JC}$, and $\mathrm{MC}$ reviewed and revised the manuscript. All authors have read and approved the final manuscript

\section{Competing interests}

The authors declare that they have no competing interests.

\section{Publisher's Note}

Springer Nature remains neutral with regard to jurisdictional claims in published maps and institutional affiliations.

\section{Author details}

${ }^{1}$ Broadcom Limited, Irvine, CA, USA. ${ }^{2}$ Department of Electrical Engineering Southern Methodist University, Dallas, TX, USA. ${ }^{3}$ Department of Computer Science, Portland State University, Portland, OR, USA. ${ }^{4}$ Cadence, Austin, TX USA. ${ }^{5}$ College of Engineering, Purdue University, West Lafayette, IN, USA.

Received: 30 July 2018 Accepted: 22 November 2018

Published online: 22 December 2018

\section{References}

1. R. Stacey, Wireless LANs. Specification framework for TGax: IEEE 802.11-15/0132r15. IEEE Standards Associations (2016)

2. Part 11: Wireless LAN medium access control and physical layer specifications. IEEE Standards Associations (2017)

3. H. Zhou, B. Li, Z. Yan, M. Yang, A channel bonding based QoS-aware OFDMA MAC protocol for the next generation WLAN. J Mob Netw Appl. 1(22), 19-29 (2017)

4. D. Bankov, A. Didenko, E. Khorov, V. Loginov, A. Lyakhov, IEEE 802.11ax uplink scheduler to minimize delay: a classic problem with new constraints. IEEE PIMRC (2017)

5. R. P. F. Hoefel, IEEE 802.11ax: joint effects of power control and iq imbalance mitigation schemes on the performance of OFDM uplink multi-user MIMO. IEEE Vehicular Technology Conference (2017)

6. G. Caire, S. Shamai, On the achievable throughput of a multiantenna Gaussian broadcast channel. Inf. Theory IEEE Trans. 49(7), 1691-1706 (2003)

7. W. Lin, B. Li, M. Yang, Q. Qu, Z. Yan, X. Zuo, B. Yang, Integrated link-system level simulation platform for the next generation wlan - IEEE 802.11ax 2016 IEEE Global Communications Conference (GLOBECOM) (2016)

8. S. Gollakota, S. D. Perli, D. Katabi, Interference alignment and cancellation. ACM SIGCOMM 2009 Conference on Data Communication (2009)

9. H. S. Rahul, S. Kumar, D. Katabi, JMB: scaling wireless capacity with user demands. ACM SIGCOMM 2012 Conference on Applications, Technologies, Architectures, and Protocols for Computer Communication (2012)

10. Y. Du, E. Aryafar, P. Cui, J. Camp, M. Chiang, SAMU: design and implementation of selectivity-aware MU-MIMO for wideband WiFi. IEEE SECON (2015)

11. Y. Du, E. Aryafar, J. Camp, M. Chiang, iBeam: intelligent client-side multi-user beamforming in wireless networks. IEEE INFOCOM (2014)

12. H. Yu, L. Zhong, A. Sabharwal, D. Kao, Beamforming on mobile devices: a first study, 17th Annual International Conference on Mobile Computing and Networking (2011)

13. E. Aryafar, M. Khojastepour, K. Sundaresan, S. Rangarajan, E. W. Knightly, Adam: an adaptive beamforming system for multicasting in wireless LANs, (Marc). IEEE INFOCOM (2012)

14. A. Goldsmith, Wireless communications, 1st edn. (University Press, Cambridge, 2005)
15. A. Bhartia, Y.-C. Chen, S. Rallapalli, L. Qiu, Harnessing frequency diversity in Wi-Fi networks. 17th Annual International Conference on Mobile Computing and Networking (2011)

16. H. K. Chung, H. L. Bertoni, Indoor propagation characteristics at $5.2 \mathrm{GHz}$ in home and office environments. Commun. Netw. J. 4(3), 176-188 (2002)

17. A. G. Siamarou, M. O. Al-Nuaimi, Wideband propagation measurements for indoor Rician fading radio channels at $62.4 \mathrm{GHz}$. Vehicular Technology Conference (2001)

18. S.-C. Kim, H. L. Bertoni, M. Stern, Pulse propagation characteristics at 2.4 GHz inside buildings. Veh. Technol. IEEE Trans. 21(1), 579-592 (1996)

19. H. L. Bertoni, Propagation effects for modern wireless systems. (Prentice hall Inc, 2000)

20. T. S. Rappaport, S. Sun, R. Mayzus, H. Zhao, Y. Azar, K. Wang, G. N. Wong, J. K Schulz, M. Samimi, F. Gutierrez, Millimeter wave mobile communications for 5 g cellular: it will work! IEEE Access. 1(1), 335-349 (2013)

21. Y. Shi, J. Wensowitch, E. Johnson, J. Camp, A measurement study of userinduced propagation effects for UHF frequency bands. IEEE International Conference on Sensing, Communication and Networking (2017)

22. H. Rahul, F. Edalat, D. Katabi, C. G. Sodini, Frequency-aware rate adaptation and MAC protocols. 15th Annual International Conference on Mobile Computing and Networking (2009)

23. S. Yun, D. Kim, L. Qiu, Fine-grained spectrum adaptation in WiFi networks. 19th Annual International Conference on Mobile Computing \& Networking (2013)

24. S. Singh, M. Shahbazi, K. Pelechrinis, K. Sundaresan, S. V. Krishnamurthy, S. Addepalli, A case for adaptive sub-carrier level power allocation in OFDMA networks. 13th ACM International Symposium on Mobile Ad Hoc Networking and Computing (2012)

25. P. Okvist, A. Simonsson, H. Asplund, LTE frequency selective scheduling performance and improvements assessed by measurements. 22nd International Symposium On Personal Indoor and Mobile Radio Communications (PIMRC) (2011)

26. The wireless open-access research platform. http://www.warpproject.org

27. F. Rusek, O. Edfors, F. Tufvesson, Indoor multi-user MIMO: measured user orthogonality and its impact on the choice of coding. 2012 6th European Conference On Antennas and Propagation (EUCAP) (2012)

28. H. Viswanathan, S. Venkatesan, H. Huang, Downlink capacity evaluation of cellular networks with known-interference cancellation. IEEE J. Sel. A. Commun. 21(5), 802-811 (2006)

29. T. Yoo, A. Goldsmith, On the optimality of multiantenna broadcast scheduling using zero-forcing beamforming. Sel. Areas Commun. IEEE J. 24(3), 528-541 (Marc)

30. T. Wu, J. Liu, T. E. Pare, Configuration signaling field and its indication. US Patent 2016/0065467 (2016)

31. V. Erceg, Indoor MIMO WLAN TGn Channel Mdels: IEEE 802.11-03/940r1. IEEE Standards Associations (2004)

\section{Submit your manuscript to a SpringerOpen ${ }^{\circ}$ journal and benefit from:}

- Convenient online submission

- Rigorous peer review

- Open access: articles freely available online

- High visibility within the field

Retaining the copyright to your article

Submit your next manuscript at $\$$ springeropen.com 